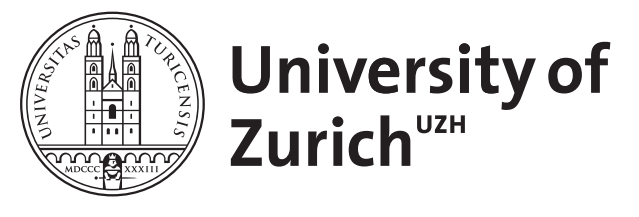

\title{
Two-dimensional infrared spectroscopy of neat ice Ih
}

\author{
Perakis, Fivos ; Hamm, Peter
}

\begin{abstract}
The $\mathrm{OH}$ stretch line shape of ice Ih exhibits distinct peaks, the assignment of which remains controversial. We address this longstanding question using two dimensional infrared (2D IR) spectroscopy of the $\mathrm{OH}$ stretch of $\mathrm{H} 2 \mathrm{O}$ and the $\mathrm{OD}$ stretch of $\mathrm{D} 2 \mathrm{O}$ of ice $\mathrm{Ih}$ at $\mathrm{T}=80 \mathrm{~K}$. The isotropic response is dominated by a $2 \mathrm{D}$ line shape component which does not depend on the pump pulse frequency. The decay time of the component that does depend on the pump frequency is calculated using singular value decomposition (bi-exponential decay H2O: 30 fs, 490 fs; D2O: 40 fs, 690 fs). The anisotropic contribution exhibits on-diagonal peaks, which decay on a very fast timescale (H2O: 85 fs; D2O: 65 fs), with no corresponding anisotropic cross-peaks. Both isotropic and anisotropic results indicate that randomization of excited dipoles occurs with a very rapid rate, just like in neat liquid water. We conclude that the underlying mechanism relates to the complex interplay between exciton migration and exciton-phonon coupling.
\end{abstract}

DOI: https://doi.org/10.1039/C2CP23710E

Posted at the Zurich Open Repository and Archive, University of Zurich

ZORA URL: https://doi.org/10.5167/uzh-64648

Journal Article

Accepted Version

Originally published at:

Perakis, Fivos; Hamm, Peter (2012). Two-dimensional infrared spectroscopy of neat ice Ih. Physical Chemistry Chemical Physics (PCCP), 14(18):6250-6256.

DOI: https://doi.org/10.1039/C2CP23710E 


\title{
Two-dimensional infrared spectroscopy of neat ice Ih
}

\author{
Fivos Perakis, Peter Hamm \\ Physikalisch-Chemisches Institut, Universität Zürich, \\ Winterthurerstrasse 190, CH-8057 Zürich, Switzerland, phamm@pci.uzh.ch
}

(Dated: December 15, 2011)

\begin{abstract}
The $\mathrm{OH}$ stretch line shape of ice Ih exhibits distinct peaks, the assignment of which remains controversial. We address this longstanding question using two dimensional infrared (2D IR) spectroscopy of the $\mathrm{OH}$ stretch of $\mathrm{H}_{2} \mathrm{O}$ and the OD stretch of $\mathrm{D}_{2} \mathrm{O}$ of ice $\mathrm{Ih}$ at $\mathrm{T}=80 \mathrm{~K}$. The isotropic response is dominated by a $2 \mathrm{D}$ line shape component which does not depend on the pump pulse frequency. The decay time of the component that does depend on the pump frequency is calculated using singular value decomposition (bi-exponential decay $\mathrm{H}_{2} \mathrm{O}: 30 \mathrm{fs}, 490 \mathrm{fs} ; \mathrm{D}_{2} \mathrm{O}: 40 \mathrm{fs}, 690 \mathrm{fs}$ ). The anisotropic contribution exhibits on-diagonal peaks, which decay on a very fast timescale $\left(\mathrm{H}_{2} \mathrm{O}\right.$ : $85 \mathrm{fs} ; \mathrm{D}_{2} \mathrm{O}: 65 \mathrm{fs}$ ), with no corresponding anisotropic cross-peaks. Both isotropic and anisotropic results indicate that randomization of excited dipoles occurs with a very rapid rate, just like in neat liquid water. We conclude that the underlying mechanism relates to the complex interplay between exciton migration and exciton-phonon coupling.
\end{abstract}

\section{INTRODUCTION}

The $\mathrm{OH}$ stretch vibrational mode is a valuable spectroscopic observable, which contains information about hydrogen bonding strength and dynamics, solvent and solute interaction, structural and dynamical heterogeneities and generally provides a direct probe to intra and intermolecular interactions. In liquid water, the $\mathrm{OH}$ stretch has been studied extensively, both with stationary and time resolved measurements [1-6]. One of the relevant features is the structure exhibited by the line shape, which becomes more prominent in the Raman spectra. In earlier interpretation, this line shape features were attributed to the symmetric and antisymmetric mode vibration, with an additional third peak attributed to the bend overtone enhanced by a Fermi resonance with the stretch [1]. Later investigations attribute the low frequency part of the $\mathrm{OH}$ stretch to strong h-bonds and the high frequency one to weak h-bonds [7], and generally this asymmetry is believed to reflect the underlying structural and dynamical inhomogeneity of liquid water [8].

Interestingly a similar debate exists for ice. Hitherto have been observed up to 15 different ice phases, which exhibit different crystal structures with respect to the oxygen positions $[9,10]$. Ice Ih is the hexagonal ice form met at atmospheric pressure bellow freezing point. The $\mathrm{OH}$ stretch mode of many of these ice forms, including ice Ih, reveals very peculiar line shapes with substructures in the form of distinct peaks, the assignment of which is still under discussion [11-19]. One of the main mechanisms suggested responsible for the line shape features, is excitonic coupling: because of inter and intramolecular coupling, the excited $\mathrm{OH}$ stretch transition dipoles add up and form delocalized intermolecular vibrations (vibrational excitons) [14, 20, 21]. Additional contributions are considered to originate from the overtone of the bending mode enhanced by a Fermi resonance with the OH stretch [22]. Finally, has been argued that excitonphonon coupling can additionally affect the line shape, i.e. the resonant $\mathrm{OH}$-stretch excitation immigration to other non-resonant excitations, such as low frequency intermolecular modes (phonons) [14].

In order to extract further information concerning the nature of these peaks, the experimental approach discussed here is to go beyond linear measurements and learn from the line shape changes under excitation, the frequency dependence of these changes and the timescale of their dynamics. So far, time resolved measurements on ice Ih focused on the isotope dilute case [23-27]. In order to study resonance effects, such as exciton coupling or vibrational energy Förster transfer [28, 29], one needs to investigate neat ice, where the excited chromophores are in resonance with the surrounding molecules. In this context, two dimensional infrared (2D IR) measurements of neat liquid water reveal a very fast initial component of $50 \mathrm{fs}$, with a corresponding anisotropy decay of $75 \mathrm{fs}$, which was attributed to energy transfer among the $\mathrm{OH}$ stretching vibrations [3].

In the present contribution, we experimentally investigate neat ice Ih $\left(100 \% \mathrm{H}_{2} \mathrm{O}\right.$ and $\left.100 \% \mathrm{D}_{2} \mathrm{O}\right)$ by $2 \mathrm{D}$ IR spectroscopy in order to provide the basis for a better understanding of the coupling mechanisms responsible for its peculiar line shape. Just like in neat liquid water [3], we observe very fast randomization of the transition dipoles that we attribute to exciton migration and finally thermalization.

\section{BACKGROUND INFORMATION: WHY 2D IR?}

2D IR spectroscopy is a powerful non-linear spectroscopic technique, which enables one to access bond specific structural resolution [30]. The combination of structural and dynamic sensitivity has been successfully applied to liquid water to extract the timescales of hydrogen bond dynamics, which manifest as a gradual loss of correlation between the pump and probe frequency [2-5]. A extension of these studies to the glassy forms of water would surely be interesting as it would allow to monitor 
the propagation of vibrational energy on a structurally disordered environment without the implication of hydrogen bond dynamics.

$2 \mathrm{D}$ IR is also sensitive to the degree of coupling between modes, which manifests itself in the $2 \mathrm{D}$ spectrum as cross-peaks between the resonances of the involved modes. For example, lets assume two coupled oscillators, the resonance frequencies of which are separated and form two distinct peaks. The 2D IR spectrum can be understood by analogy with a double-resonance pumpprobe experiment, where we can acquire the frequency dependence of both pump and probe pulses. When both pump and probe are resonant with one of the oscillators, the resulting $2 \mathrm{D}$ IR peak is diagonal (i.e. has the same frequency for pump and probe), providing information on the dynamics of this state. In the case when the pump excites the one peak, and the probe is resonant with the other, the resulting $2 \mathrm{D}$ peak is a cross-peak, which can be used to calculate the coupling strength between the states.

More generally speaking, one can learn about the the degree of connectivity between two (or more) different quantum states by a double-resonant excitation to a mixed state, which contains additional information than 1D spectroscopy, where the direct excitation to such mixed states is dark. Besides excitonic coupling (which is the coupling between two almost harmonic modes that are close in resonance) discussed above, on can investigate other intrinsically anharmonic coupling mechanisms, such as Fermi resonances or exciton-phonon coupling. 2D IR spectroscopy does in principle provide the information needed to disentangle such competing coupling mechanisms, in contrast to $1 \mathrm{D}$ spectroscopy. For example, in Ref. [31] it has been shown that a 2D spectrum can discriminate a Fermi resonance from polaronic effects, despite he fact that the corresponding 1D spectra looked almost identical.

Polarization control is an additional feature in 2D IR spectroscopy that can be used to measure the angle between pumped and probed transition dipole [30]. To demonstrate this principle, lets assume that both pump and probe pulses have the same polarization $S_{Z Z Z Z}$. When the population time $t_{2}$ (i.e. the waiting time between pump and probe) is 0 , the excited transition dipole by the pump has the same direction when probed (the angle between them is 0). Giving the system some time to evolve $\left(t_{2}>0\right)$ the excitation can immigrate to other modes (for example due to excitonic coupling) and as a result the memory of the direction of the excitation vanishes. This can still give signal in the $S_{Z Z Z Z}$ polarization geometry as well as in the $S_{Z Z Y Y}$. However taking the anisotropic signal $\left(S_{Z Z Z Z}-S_{Z Z Y Y}\right)$ one can acquire information only from the directly excited states which are directionally dependent, whereas the isotropic signal $\left(S_{Z Z Z Z}+S_{Z Z Y Y}\right) / 3$ provides information about the in-directly excited states (i.e. those excited later by immigration of the excitation or thermalization) which are directionally independent.

\section{MATERIALS AND METHODS}

The laser system used for the 2D IR measurements is a commercial Ti:Sapphire oscillator and chirped pulse amplifier with repetition rate of $1 \mathrm{kHz}$, giving pulses centered around $800 \mathrm{~nm}$ of energy $1 \mathrm{~mJ}$. An home-built optical parametric amplifier (OPA) [32] was used to generate tunable mid-IR pulses of energy $2 \mu \mathrm{J}$, bandwidth $300 \mathrm{~cm}^{-1}$ and duration $\simeq 65 \mathrm{fs}$ (estimated by interferometric autocorrelation trace and compressed with $\mathrm{CaF}_{2}$ windows [33]). For the OD stretch measurements the pulses were centered at $2320 \mathrm{~cm}^{-1}$ and for the $\mathrm{OH}$ at $3200 \mathrm{~cm}^{-1}$.

The 2D spectra were recorded using a Fourier transform 2D IR setup in pump-probe geometry [34, 35], where the data acquisition is performed with a fast scanning routine, described in detail elsewhere [35]. In brief, the mid-IR beam is split to two parts: the pump and the probe. The pump pulse goes through an interferometer, where the time delay between the two arms is refereed to as coherence time $t_{1}$; consequently a second time delay modulates the pump with respect to the probe pulse, referred here as population time $t_{2}$. We scanned $t_{1}=(-300,900)$ fs and recorded spectra for $t_{2}=(-100,1000)$ fs with a step of 10 fs. All samples were measured with $S_{Z Z Z Z}$ and $S_{Z Z Y Y}$ polarization geometries; to interchange between the two we used a $\lambda / 2$ waveplate mounted on a custom-made computercontrolled base, to flip the pump polarization between successive scans. A wobbling Brewster window was used for scattering suppression (by phase modulation) $[35,36]$

All the measurements were performed at $T=80 \mathrm{~K}$ using a nitrogen cooled cryostat (open cycle) and using as samples triple distilled $\mathrm{H}_{2} \mathrm{O}$ and $\mathrm{D}_{2} \mathrm{O}$ (Sigma Aldrich $>99.9 \%$ ). The sample cell was constructed in nitrogen chamber to maintain the purity of the $\mathrm{D}_{2} \mathrm{O}$, which was pressed against two $\mathrm{CaF}_{2}$ windows without spacer and using just the surface tension to keep the sample together (estimated thickness $\sim 0.5 \mu \mathrm{m}$ ).

\section{EXPERIMENTAL RESULTS}

\section{A. Linear absorbance (FTIR) spectra}

We refer to stationary (FTIR) spectra as linear (with respect to the number of field interactions with the sample) to discriminate from nonlinear methods (pumpprobe, 2D IR). In fig. 1 is shown the linear absorbance spectra of ice Ih, for: (a) the $\mathrm{OH}$ stretch of $\mathrm{H}_{2} \mathrm{O}$ (blue) and $\mathrm{HOD} / \mathrm{D}_{2} \mathrm{O}$ (red) and (b) for the OD stretch of $\mathrm{D}_{2} \mathrm{O}$ (blue) and $\mathrm{HOD} / \mathrm{H}_{2} \mathrm{O}$ (red). To facilitate the comparison between the two, the spectra are normalized to the maximum amplitude of the ice peaks, and in the case of isotope diluted samples, a constant offset has been subtracted to partially account for the background absorption of the major component (e.g. the $\mathrm{H}_{2} \mathrm{O}$ absorbance in the OD region of the $\mathrm{HOD} / \mathrm{H}_{2} \mathrm{O}$ case). 


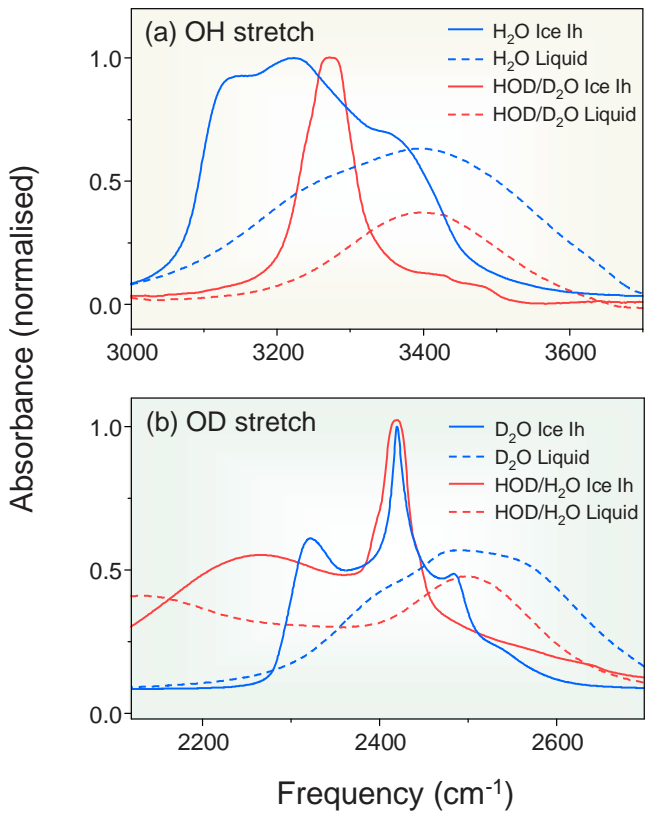

FIG. 1: FTIR spectra of the (a) OH stretch mode of $\mathrm{H}_{2} \mathrm{O}$ (blue) and of HOD in $\mathrm{D}_{2} \mathrm{O}$ (red) and the (b) OD stretch mode of $\mathrm{D}_{2} \mathrm{O}$ (blue) and $\mathrm{HOD} / \mathrm{H}_{2} \mathrm{O}$ (red). The spectra of liquid water (dashed lines) appear blue-shifted and broader than that of ice Ih (solid lines). The isotope dilute case is much more narrow than that of the neat ice, which exhibits substructure and distinct peaks.

The spectra of ice (solid lines) appear red-shifted from that of the liquid (dashed lines), which reflect the transition to a stronger hydrogen bonded environment and shorter bond length [37]. The broad line shape of the $\mathrm{OH}$ stretch in liquid water is typically assigned to inhomogeneity due to different hydrogen bonded environments around the $\mathrm{OH}$ (or OD) chromophore [8].The $\mathrm{OH}$ stretch line shape of the isotope dilute ice $5 \% \mathrm{HOD}$ in $\mathrm{D}_{2} \mathrm{O}$ (and $5 \% \mathrm{HOD}$ in $\mathrm{H}_{2} \mathrm{O}$ for the $\mathrm{OD}$ ) is significantly more narrow. This feature reflects the more ordered environment (no hydrogen bond dynamics), as well as the lack of intermolecular coupling between the local chromophore (for the $\mathrm{OH}$ stretch) with the surrounding isotopic modes (the OD stretch and vice versa) [38]. On the contrary, in the case of neat ice $\left(100 \% \mathrm{H}_{2} \mathrm{O}\right.$ and $\left.100 \% \mathrm{D}_{2} \mathrm{O}\right)$ after freezing the band remains broad and furthermore develops distinct substructure with decreasing temperature. The broad band observed in the $\mathrm{OD}$ stretch of $\mathrm{HOD} / \mathrm{H}_{2} \mathrm{O}$ is the combination band of the bend with the librations and originates from the water background.

Our spectra agree with those found in literature [11$16]$, setting the stage for the main topic: the two dimensional infrared results.

\section{B. Two dimensional infrared (2D IR) spectra}

\section{General description}

A selection of 2D IR spectra of neat (100\%) $\mathrm{H}_{2} \mathrm{O}$ and $\mathrm{D}_{2} \mathrm{O}$ ice Ih at $T=80 \mathrm{~K}$ are shown in figures 2 and 3 , respectively. The pump frequency $\omega_{\text {pump }}$ is depicted by convention on the vertical axis, and the probe $\omega_{\text {probe }}$ on the horizontal. The blue lobes are typically associated with ground state processes (bleaching and stimulated emission) and the red ones, with inverted sign, with those with the excited state (excited state absorption). In this particular case however, this statement is not necessarily true, since there can be mutual cancelations from the different overlapping bands as well as additional thermal contributions.

\section{Isotropic signal $\left(S_{Z Z Z Z}+2 S_{Z Z Y Y}\right) / 3$}

In the isotropic spectra we observe that the line shape is elongated along the pump axis, which for later population times becomes practically independent of the pump frequency (fig.2,3:middle row), apart from an overall intensity that reflects the linear absorption spectrum. That is, for late times, pump and probe frequency are uncorrelated, so that the $2 \mathrm{D}$ response takes the form:

$$
S_{2 D}\left(\omega_{\text {pump }}, \omega_{\text {probe }}\right)=S_{1 D}\left(\omega_{\text {pump }}\right) \times S_{1 D}^{\prime}\left(\omega_{\text {probe }}\right)
$$

where $S_{1 D}\left(\omega_{\text {pump }}\right)$ reflects the amount of absorbed light, and $S_{1 D}^{\prime}\left(\omega_{\text {probe }}\right)$ would be the $1 \mathrm{D}$ pump-probe response. Any pump frequency dependence, that is present in the $2 \mathrm{D}$ spectra at $t_{2}=150 \mathrm{fs}$ to $300 \mathrm{fs}$, decays very quickly. We extract the timescales of it using singular value decomposition (SVD) analysis, as as discussed further down.

A red lobe is observed for lower probe frequencies (Figs. 2,3: label I), which is attributed to the excited state absorbance, the broad line shape of which is understood from the simpler isotope diluted case: it was shown to be due to the anharmonic dependence of the vibrational potential from the $\mathrm{R}_{\mathrm{O}} \ldots \mathrm{O}$ distance, combined with coupling to the crystal phonons [27]. The observation of similar spectral characteristics between the two cases (neat and isotope diluted) allows us to conclude that the same mechanism is present to some extend here. One important difference, however, between the isotope dilute and the neat case, is that the excitation is localized in the first (because isolated chromophore is off resonance), whereas delocalized in the latter (due to intermolecular coupling). Because of excitonic coupling, only a small part of the excitation remains localized as in the isotope dilute case. This may explain why the broad excited state band (label I) is not as pronounce here as in the isotope dilute case.

For later population times, a feature shows up at higher probe frequencies (label II), which has been attributed to weakening of the hydrogen bonds upon heating causing a 


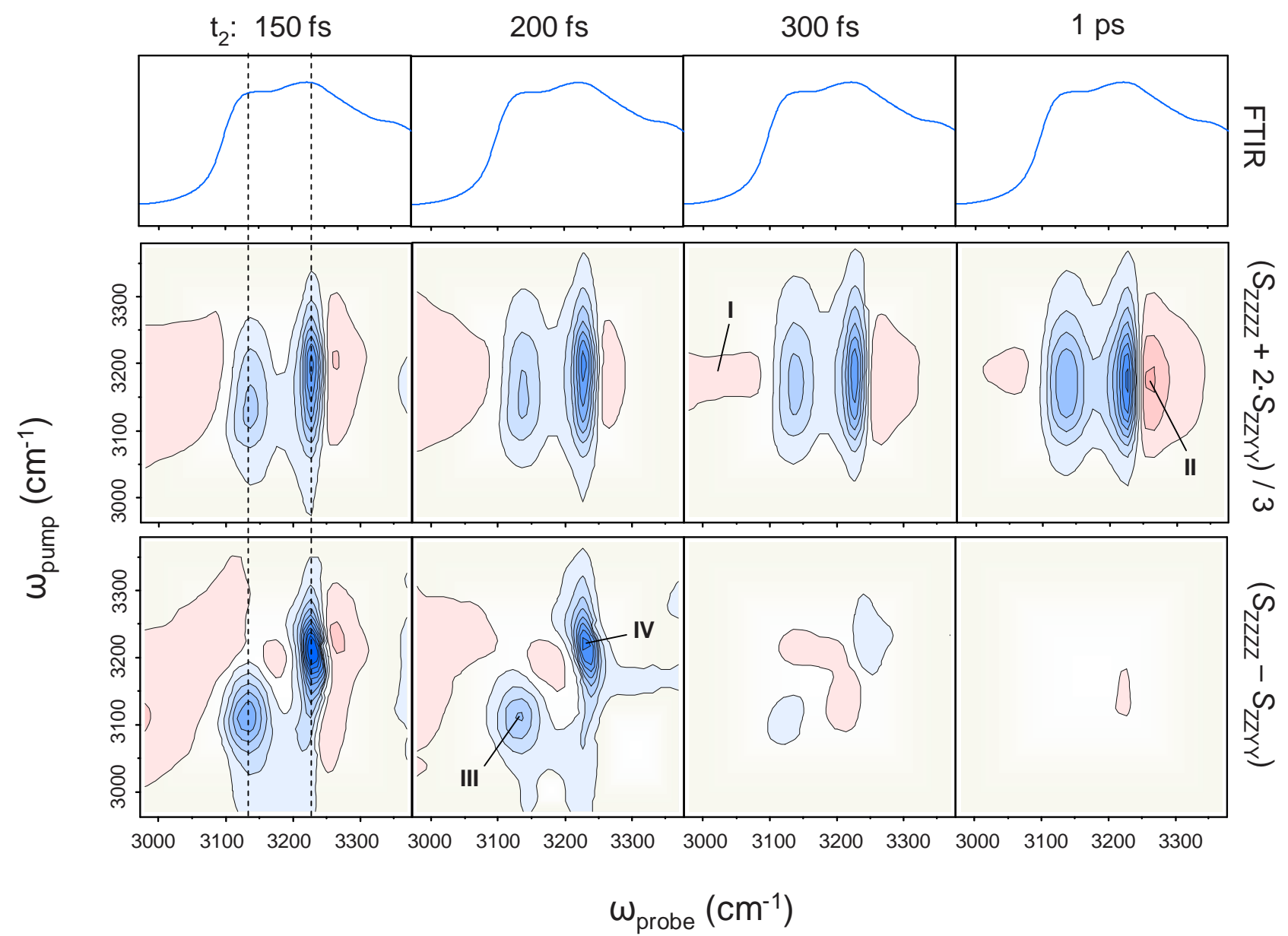

FIG. 2: Purely absorptive 2D IR spectra of $\mathrm{H}_{2} \mathrm{O}$ ice Ih at $T=80 \mathrm{~K}$. By convention we depict the vertically the pump frequency axis $\omega_{\text {pump }}$ and horizontally the probe $\omega_{\text {probe }}$. Upper row: linear spectra in the same frequency range as the $\omega_{\text {probe }}$. Middle row: Isotropic spectra $\left(S_{Z Z Z Z}+2 \cdot S_{Z Z Y Y}\right) / 3$. Lower row: Anisotropic spectra $\left(S_{Z Z Z Z}-S_{Z Z Y Y}\right)$. In the different columns are displayed the different population times $\mathrm{t}_{2}$ as indicated. The labels I-IV indicate line shape features discussed in the text.

blue shift of the $\mathrm{OH}$ stretch absorption. A similar signal is also observed in liquid [4] and supercooled water [5], as well as in isotope dilute ice [25-27]. However, we note that the sample cannot be fully thermalized after $1 \mathrm{ps}$, since the $2 \mathrm{D}$ response evolves in time along the probe frequency axis (see below).

\section{Anisotropic signal $\left(S_{Z Z Z Z}-S_{Z Z Y Y}\right)$}

In the case of anisotropic spectra $\left(S_{Z Z Z Z}-S_{Z Z Y Y}\right)$, the line shape exhibits distinct features in the form of ondiagonal peaks, as can be seen from Figs.2,3 (lower row). The extracted signal reveals response for three peaks in the case of the $\mathrm{D}_{2} \mathrm{O}$, in agreement with the frequency observed from the linear spectra (fig.3, labels III,IV,V), whereas only two in the case of $\mathrm{H}_{2} \mathrm{O}$ (fig. 2, labels II and
III). The third peak is completely absent even when the experiment was repeated with pump frequency centered around this peak. A similar observation has been made for crystalline acetanilide (ACN) [31], another hydrogenbonded crystal, where it was attributed to delocalization of the state that renders it more harmonic.

An additional observation is that the line shape of the lower frequency peak (label III) is somehow rounder than that of the high frequency ones (labels IV,V), which appear more elongated, indicating possibly a different underlying physical mechanism. In the linear spectra, these peaks were attributed to strong and weak intermolecular coupling by mixed classic/QM simulations [21], a assignment which could result to different $2 \mathrm{D}$ line shapes, like the ones observed here. Finally, we note that we do not observe any anisotropic cross-peaks between the observed peaks. 


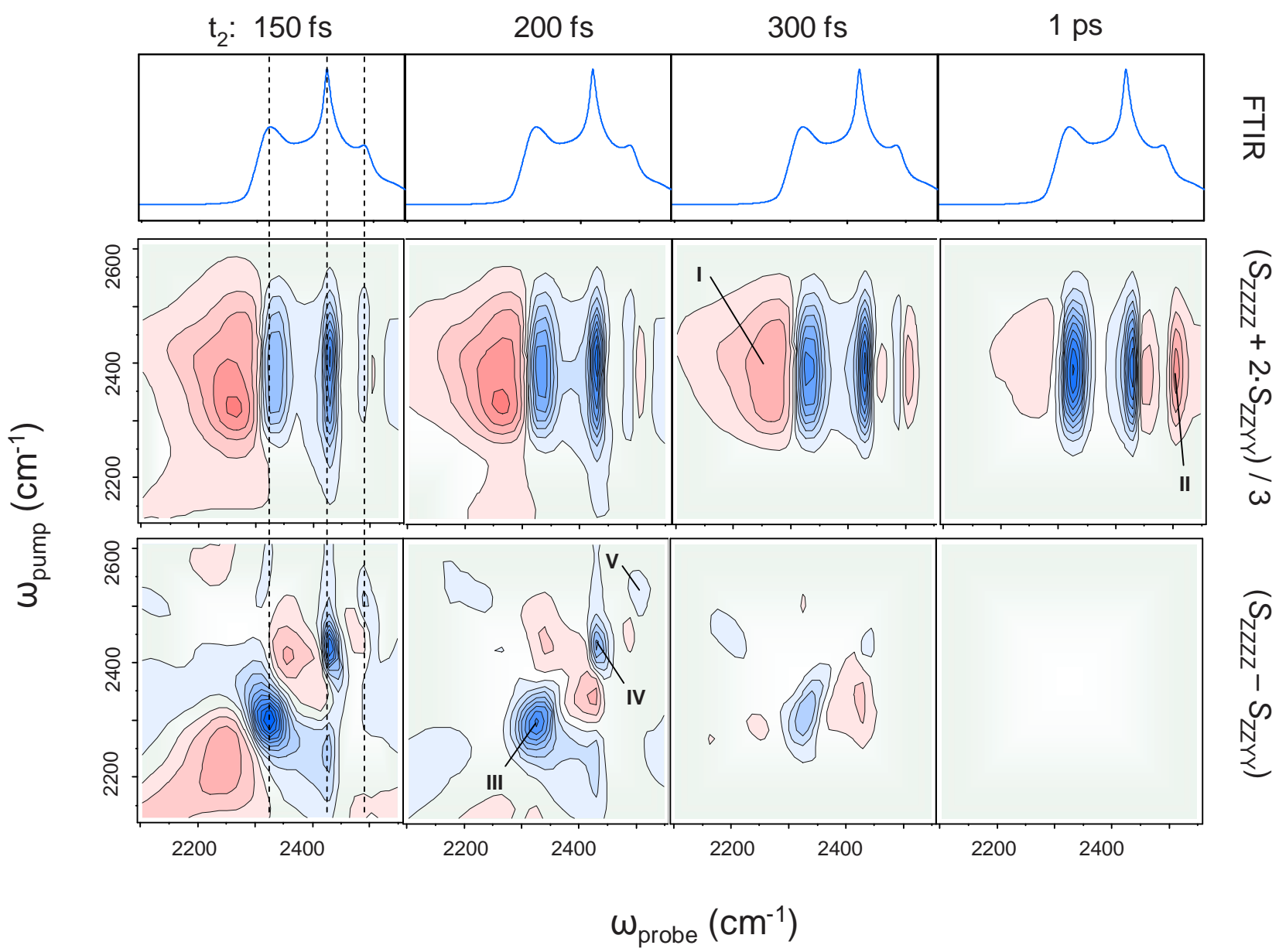

FIG. 3: Purely absorptive 2D IR spectra of $\mathrm{D}_{2} \mathrm{O}$ ice Ih at $T=80 \mathrm{~K}$. The figure is structured just like fig.2

\section{Dynamics}

The dynamics of the observed line shape features are qualitatively the same for both $\mathrm{H}_{2} \mathrm{O}$ and $\mathrm{D}_{2} \mathrm{O}$. In fig. 4 is shown the time dependence of the lower frequency peak (fig.2,3: label III). The choice of the particular peak is arbitrary, since all peaks exhibit qualitatively similar dynamics. Here, each point represents one 2D IR spectrum.

As discussed already, the isotropic signal $\left(S_{Z Z Z Z}+\right.$ $\left.2 S_{Z Z Y Y}\right) / 3$ (fig. 4, green circles) does not decay to zero. The anisotropic signal $\left(S_{Z Z Z Z}-S_{Z Z Y Y}\right)$, in contrast decays completely on a very fast timescale (fig. 4, orange squares); exponential fits reveal decay constants of 85 fs for the $\mathrm{H}_{2} \mathrm{O}$, and 65 fs for the $\mathrm{D}_{2} \mathrm{O}$. The anisotropy decay reflects the randomization of the direction of the excited transition dipoles. No quantum beating is resolved within the signal to noise ratio, like it was in isotope diluted ice [27].
5. Singular Value Decomposition (SVD)

The isotropic signal is dominated by contributions which, at least for later times, do not seem to depend on the pump frequency. Just like the anisotropy decay, this is another measure of the degree of randomization of the excitation. The timescales of this randomization can be extracted using singular value decomposition (SVD) [39], which is based on the theorem that any matrix $S$ can be written as :

$$
S=U \cdot \operatorname{diag}\left[w_{i}\right] \cdot V^{T}
$$

where $U$ and $V$ are each column-orthogonal matrices and $w_{i}$ are the singular values. In other words, SVD decomposes the matrix $S$ into an orthonormal set of basis vectors (columns of $U$ ) which correspond to individual singular values $w_{i}$.

Considering matrix $S$ to be the $2 \mathrm{D}$ response $S\left(\omega_{\text {pump }}, \omega_{\text {probe }}\right)$, SVD leads to a matrix $U$, the columns 


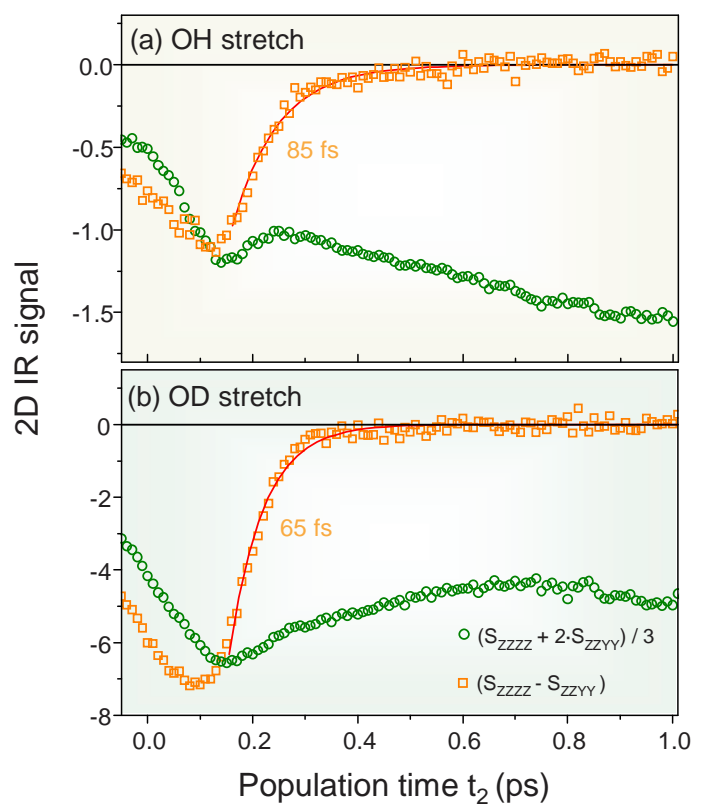

FIG. 4: 2D IR signal dynamics of the (a) $\mathrm{OH}$ stretch of $\mathrm{H}_{2} \mathrm{O}$ and (b) OD stretch of $\mathrm{D}_{2} \mathrm{O}$ of neat ice Ih. The different peaks of the $2 \mathrm{D}$ IR difference spectrum exhibit quantitatively similar dynamics; here we plot the signal decay for the lower frequency peak (Fig.2,3: label III). Horizontally is depicted the population time $t_{2}$ and vertically the signal size for the isotropic (green circles) and anisotropic (orange squares) contributions.

of which correspond to spectra as a function of pump frequencies $\omega_{\text {pump }}$ and same for $V$ for the probe frequencies $\omega_{\text {probe }}$. These spectra are normalized, and the amplitudes are given by the corresponding singular values $w_{i}$. We have seen that in the isotropic case the $2 \mathrm{D}$ line shapes become pump-frequency independent at later times (Eq. 1). In the extreme case where the line shape is completely independent of the pump frequency, all singular values will be zero (within signal-to-noise), except from one. The elements of the corresponding $2 \mathrm{D}$ response would then be:

$$
s_{n m}=u_{n, 1} \cdot w_{1} \cdot v_{m, 1}
$$

where $u_{n, 1}$ relates to $S_{1 D}\left(\omega_{\text {pump }}\right)$ and $v_{m, 1}$ to $S_{1 D}^{\prime}\left(\omega_{\text {probe }}\right)$ as seen on equation 1 .

At early times, the 2D line shape does partially depend on the pump frequency, revealing more than one non-zero singular values $w_{i}$. If $w_{1}$ is the largest singular value, dividing with $\sqrt{\sum w_{i}^{2}}$ normalizes $w_{1}$ over all the singular values. Then, the quantity:

$$
\sigma_{s v d}=1-\frac{w_{1}}{\sqrt{\sum w_{i}^{2}}}
$$

is a measure of the pump-frequency dependence. Its decay with population time $t_{2}$ is shown in Fig.6. Both isotopomers exhibit a bi-exponential decay with a fast component $\left(\mathrm{H}_{2} \mathrm{O}: 30 \mathrm{fs}, \mathrm{D}_{2} \mathrm{O}: 40 \mathrm{fs}\right)$ which is on the same

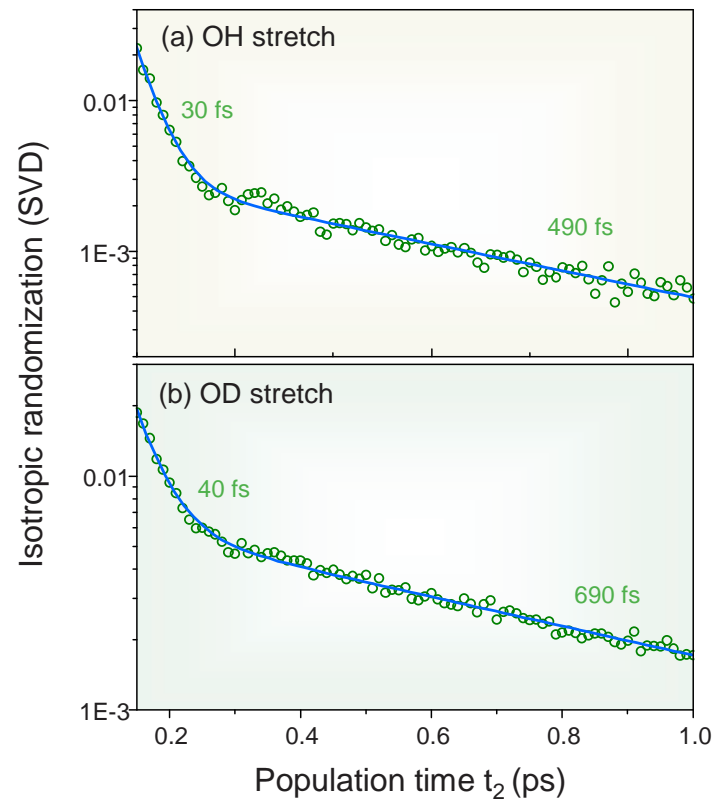

FIG. 5: The dependence of the isotropic 2D IR line shape on the pump frequency, can be calculated using SVD (see text). This provides a measure of the transition dipole randomization. Here is shown the $\sigma_{s v d}$ as a function of population time for both the (a) $\mathrm{OH}$ stretch of $\mathrm{H}_{2} \mathrm{O}$ and (b) OD stretch of $\mathrm{D}_{2} \mathrm{O}$ of neat ice Ih.

order like that of the the anisotropy decay dynamics, consistent with the statement that both are complementary measures of randomization. Additionally, we note a slower component that is evident for both isotopomers $\left(\mathrm{H}_{2} \mathrm{O}: 490\right.$ fs, $\left.\mathrm{D}_{2} \mathrm{O}: 690 \mathrm{fs}\right)$.

\section{CONCLUSIONS AND SUMMARY}

In this contribution, we describe the experimental results of the 2D IR spectroscopic investigation of neat ice Ih, for both $\mathrm{H}_{2} \mathrm{O}$ and $\mathrm{D}_{2} \mathrm{O}$. We performed measurements for both polarizations $S_{Z Z Z Z}$ and $S_{Z Z Y Y}$ and our analysis focuses on the early sub-picosecond dynamics of the isotropic and the anisotropic signals. Our main conclusions are summarized below:

- We observe very fast randomization of the transition dipoles, which exhibits both isotropic and anisotropic components, in agreement with $2 \mathrm{D}$ IR measurements of neat liquid water [3]. One possible mechanism for randomization of transitions dipoles is exciton migration, a assignment which seems proper when one considers excitonic coupling as the origin of the observed bands [21]. The observed timescales agree with isotope concentration dependent pump-probe measurements on ice Ih, where the fast anisotropy decay component (140 fs) 
was attributed to vibrational Förster energy transfer [28]. Another possible mechanism responsible for anisotropy decay would be reorientation of the water molecules, which however in ice Ih is expected on a nano- to micro-second timescale[40, 41].

- In the isotropic response, randomization affects the 2D line shape resulting in spectra that do not depend to the pump frequency. The timescales of this process are extracted by singular value decomposition (SVD), which reveals a bi-exponential decay for both isotopomers $\left(\mathrm{H}_{2} \mathrm{O}: 30 \mathrm{fs}, 490 \mathrm{fs} ; \mathrm{D}_{2} \mathrm{O}: 40 \mathrm{fs}\right.$, $690 \mathrm{fs})$.

- The anisotropic response reveals diagonal peaks (fig. 2,3: label III-V), which exhibit a variety of line shapes for both isotopomers. Their very fast decay provides another measure of randomization on a sub 100 fs timescale $\left(\mathrm{H}_{2} \mathrm{O}: 85 \mathrm{fs} ; \mathrm{D}_{2} \mathrm{O}: 65 \mathrm{fs}\right)$.

- Because of the multiple decaying timescales observed it becomes difficult to clearly assign what is vibrational relaxation time $T_{1}$ of the $\mathrm{OH}$ vibration of neat ice Ih. This difficulty might not only be on an experimental (i.e., fitting properly the data), but also on a conceptual level. That is, if the coupling between the $\mathrm{OH}$ vibration and other degrees of freedom such as crystal phonons is very strong, a perturbative treatment leading to a $T_{1}$ relaxation rate might no longer be adequate. Rather, one might have to think about the excitation more in the sense of a wave packet evolving on a coupled, highly anharmonic potential energy surface.

- We do not observe any cross-peaks in the anisotropic 2D spectra, which suggests that the two corresponding bands are not related to excitonic splitting (which should in fact reveal a strong cross peak). This conclusion seems to contradict the observation that the excitation is randomized on a very fast time scale, presumably by exciton migration. This discrepancy remains to be resolved.

- Additionally, line shape analysis of the isotropic signal reveals contributions from exciton-phonon coupling (fig. 2,3: label I), in agreement with those observed for isotope diluted ice Ih [27]. Possibly, due to the delocalization of the excitation in the neat case [21], the effect is much weaker. Consistent with this interpretation, no quantum beating related with the crystal phonons [27] are resolved within the signal-to-noise ratio of the measurement.

- Lastly, the isotropic dynamics exhibit a picosecond timescale rise, just like those observed from tran- sient grating measurements of neat liquid water [3], where it was attributed to heating effects associated with vibrational relaxation. Interestingly, the dynamics in ice Ih exhibit evolution beyond 1 ps, similar to those recorded when superheating and melting of isotope dilute ice Ih [25]. We will address this observation on a following publication.

Explaining the $\mathrm{OH}$ spectra of hydrogen-bonded molecular crystals is a notoriously difficult problem, and ice is one of these cases. Potentially, three coupling mechanisms might be responsible for the peculiar $1 \mathrm{D}$ and $2 \mathrm{D}$ line shapes of neat ice: (i) intra- and inter-molecular excitonic coupling of the $\mathrm{OH}$ groups, which is a result of the close packing and the large transition dipole, (ii) coupling to crystal phonons (i.e., vibrational polarons), which reflects the large anharmonicity of the $-\mathrm{OH} \cdots \mathrm{O}$ unit, and (iii) a Fermi-resonance with the $\mathrm{OH}$ bending mode which happens to be at pretty much exactly half the frequency of the $\mathrm{OH}$ stretch. Very likely, the couplings of all these mechanisms are of comparable strength, as such it is impossible to single out one. Augmented with the proton disorder that breaks the translational symmetry of the crystal, this becomes a truly challenging problem from a theoretical point of view. The model of Ref. [21] explicitly included exciton coupling on a fully quantum level, the exciton-phonon coupling on a mixed quantumclassical level, but did not include any Fermi-resonance. It gave reasonable agreement for the $1 \mathrm{D}$ absorption spectrum of neat ice Ih. Conceptually speaking, the extension of the model to $2 \mathrm{D}$ spectroscopy should be feasible, however, it might be very demanding with regard to computer resources. It will be interesting to see whether different crystalline forms of ice, where the coupling patterns are different, lead to different 2D responses that furthermore can be understood theoretically. We would like to stress again that $2 \mathrm{D}$-IR does in principle provide the information needed to disentangle such coupling mechanisms, in contrast to $1 \mathrm{D}$ spectroscopy. We therefore very much hope that our experimental results can initiate theoretical efforts in this direction.

\section{ACKNOWLEDGMENTS}

This work has been initiated by an inspiring discussion with Jim Skinner. We would like to thank Dr. Jan Helbing and Julien Réhault for their continuous help in the lab. This work was supported by the Swiss National Science Foundation (SNF) through the NCCR MUST.
[1] M. Falk and T. Ford, Can. J. Chem., 1966, 44, 16991707.
[2] J. B. Asbury, T. Steinel, K. Kwak, S. A. Corcelli, C. P. 
Lawrence, J. L. Skinner, and M. D. Fayer, J. Chem. Phys., 2004, 121, 12431-12446.

[3] M. L. Cowan, B. D. Bruner, N. Huse, J. R. Dwyer, B. Chugh, E. T. J. Nibbering, T. Elsaesser, and R. J. D. Miller, Nature, 2005, 434, 199-202.

[4] R. A. Nicodemus, K. Ramasesha, S. T. Roberts, and A. Tokmakoff, J. Phys. Chem. Let., 2010, 1, 1068-1072.

[5] F. Perakis and P. Hamm, J. Phys. Chem. B, 2011, 115, 5289-5293.

[6] S. Garrett-Roe, F. Perakis, F. Rao, and P. Hamm, J. Phys. Chem. B, 2011, p. 6976.

[7] S. T. Roberts, K. Ramasesha, and A. Tokmakoff, Acc. Chem. Res., 2009, 42, 1239-1249.

[8] S. Garrett-Roe and P. Hamm, Phys. Chem. Chem. Phys., 2010, 12, 11263.

[9] E. A. Zheligovskaya and G. G. Malenkov, Russ. Chem. Rev., 2006, 75, 57-76.

[10] C. G. Salzmann, P. G. Radaelli, E. Mayer, and J. L. Finney, Phys. Rev. Lett., 2009, 103, 105701-4.

[11] C. Haas and D. F. Hornig, J. Chem. Phys., 1960, 32, 1763.

[12] J. E. Bertie and E. Whalley, J. Chem. Phys., 1964, 40, 1637.

[13] J. E. Bertie, J. Chem. Phys., 1969, 50(10), 4501.

[14] E. Whalley, Can. J. Chem., 1977, 55, 3429-3441.

[15] J. R. Scherer and R. G. Snyder, J. Chem. Phys., 1977, 67, 4794 .

[16] M. S. Bergren, D. Schuh, M. G. Sceats, and S. A. Rice, J. Chem. Phys., 1978, 69, 3477.

[17] J. E. Bertie and E. Whalley, J. Chem. Phys., 1964, 40, 1646.

[18] J. E. Bertie, J. Chem. Phys., 1968, 49, 2141.

[19] J. E. Bertie and F. E. Bates, J. Chem. Phys., 1977, 67, 1511.

[20] V. Buch and J. P. Devlin, J. Chem. Phys., 1999, 110, 3437.

[21] F. Li and J. L. Skinner, J. Chem. Phys., 2010, 133, 244504.

[22] S. A. Rice, M. S. Bergren, A. C. Belch, and G. Nielsen, J. Phys. Chem., 1983, 87, 4295-4308.

[23] G. Seifert, K. Weidlich, and H. Graener, Phys. Rev. B, 1997, 56, R14231.

[24] S. Woutersen, U. Emmerichs, H. Nienhuys, and H. J. Bakker, Phys. Rev. Lett., 1998, 81, 1106.

[25] H. Iglev, M. Schmeisser, K. Simeonidis, A. Thaller, and A. Laubereau, Nature, 2006, 439, 183-186.

[26] A. M. Dokter and H. J. Bakker, J. Chem. Phys., 2008, 128, 024502.

[27] F. Perakis, S. Widmer, and P. Hamm, J. Chem. Phys., 2011, 134, 204505.

[28] R. L. A. Timmer and H. J. Bakker, J. Phys. Chem. A, 2010, 114, 4148-4155.

[29] M. Yang, F. Li, and J. L. Skinner, J. Chem. Phys., 2011, 135, 164505.

[30] P. Hamm and M. T. Zanni, Concepts and Methods of 2D Infrared Spectrsocopy, Cambridge University Press, Cambridge, 2011.

[31] J. Edler and P. Hamm, J. Chem. Phys., 2003, 119, 2709.

[32] P. Hamm, R. A. Kaindl, and J. Stenger, Opt. Lett., 2000, 25, 1798.

[33] N. Demirdoven, M. Khalil, O. Golonzka, and A. Tokmakoff, Opt. Lett., 2002, 27(6), 433-435.

[34] L. P. DeFlores, R. A. Nicodemus, and A. Tokmakoff, Opt. Lett., 2007, 32, 2966-2968.
[35] J. Helbing and P. Hamm, J. Opt. Soc. Am. B, 2011, 28(1), 171-178.

[36] R. Bloem, S. Garrett-Roe, H. Strzalka, P. Hamm, and P. Donaldson, Opt. Express, 2010, 18, 27067-27078.

[37] S. A. Corcelli and J. L. Skinner, J. Phys. Chem. A, 2005, 109, 6154 .

[38] F. Li and J. L. Skinner, J. Chem. Phys., 2010, 132, 204505.

[39] W. T. V. B. P. F. W. H. Press, S. A. Teukolsky, Numerical recipes in $C$, Cambridge University Press, Cambridge, 1992.

[40] M. Kunst and J. M. Warman, J. Phys. Chem., 1983, 87, 4093-4095.

[41] B. Geil, T. M. Kirschgen, and F. Fujara, Phys. Rev. B, 2005, 72, 014304. 


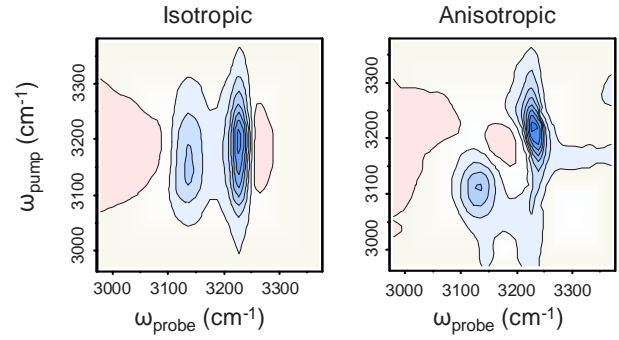

FIG. 6: TOC Graphics: Isotropic and anisotropic 2D-IR spectrum of neat ice Ih, reporting on the couplings that give rise to its peculiar lineshape. 